\title{
Le Bickenberg à Osenbach (Haut-Rhin)
}

Etude d'un paysage d'enclos médiéval sur les collines calcaires sèches sous-vosgiennes

The Bickenberg at Osenbach (Haut-Rhin, France). Study of a medieval enclosure landscape on the xeric calcareous hills of the piedmont of the Vosges Der Bickenberg bei Osenbach (Haut-Rhin, Frankreich). Untersuchung von mittelalterlichen Feldeinfassungen auf xerophilen Kalkhügeln in der Vorbergzone der Vogesen

Dominique Schwartz, Laurence Bernardy, Claire Jouve-Litaudon et Anne Vonna

\section{OpenEdition} Journals

Édition électronique

URL : http://journals.openedition.org/rge/2340

DOI : $10.4000 /$ rge. 2340

ISSN : 2108-6478

Éditeur

Association des géographes de l'Est

Édition imprimée

Date de publication : 1 juin 2003

ISSN : 0035-3213

Référence électronique

Dominique Schwartz, Laurence Bernardy, Claire Jouve-Litaudon et Anne Vonna, «Le Bickenberg à Osenbach (Haut-Rhin) », Revue Géographique de l'Est [En ligne], vol 43 / 3 | 2003, mis en ligne le 25 novembre 2010, consulté le 08 septembre 2020. URL : http://journals.openedition.org/rge/2340 DOI : https://doi.org/10.4000/rge.2340

Ce document a été généré automatiquement le 8 septembre 2020.

Tous droits réservés 


\section{Le Bickenberg à Osenbach (Haut- Rhin)}

Etude d'un paysage d'enclos médiéval sur les collines calcaires sèches sous-vosgiennes

The Bickenberg at Osenbach (Haut-Rhin, France). Study of a medieval enclosure landscape on the xeric calcareous hills of the piedmont of the Vosges

Der Bickenberg bei Osenbach (Haut-Rhin, Frankreich). Untersuchung von mittelalterlichen Feldeinfassungen auf xerophilen Kalkhügeln in der Vorbergzone der Vogesen

Dominique Schwartz, Laurence Bernardy, Claire Jouve-Litaudon et Anne Vonna

\section{Introduction}

Contrairement à d'autres régions françaises, l'archéologie des paysages agraires n'a pas connu de réel développement récent en Alsace. La plupart des travaux concernant cette région sont anciens, et se sont focalisés sur la plaine rhénane : étude des centuriations et voies romaines (Hatt, 1959; Juillard, 1959; Biehler, 1965; Wurtz, 1976), de l'occupation du sol et des activités agricoles, de l'openfield et de ses structures caractéristiques: crêtes de labour (Ackerberge), laniérage prononcé du parcellaire (Sittig, 1934; Juillard, 1953a et b; Boehler, 1967; Callot, 1980). Dans le secteur montagnard, le travail de référence de Boyé (1903) a eu peu de suite. L'étude par Koerner et al. (1999a et b) de l'influence des pratiques agricoles anciennes des Anabaptistes sur la dynamique actuelle de forêts vosgiennes fait exception avec les travaux historiques de Garnier (1993, 1998, 2000, 2002). Quant aux collines sousvosgiennes, les quelques travaux consacrés à cette zone se sont focalisés sur l'histoire de la viticulture et ne traitent pas non plus de ces questions (Hertzog, 1900 ; Dion, 1959 ; Sittler et Marocke, 1981). 
2 Ce secteur a sans doute été le moins étudié, alors qu'étrangement, il abrite localement des structures agraires tout à fait originales pour l'Alsace. En particulier, un véritable "bocage pierrier" se développe sur quelques collines sous-vosgiennes à l'ouest de Rouffach. A notre connaissance, aucune publication, ni même de description sommaire n'a été consacrée à ces structures lithiques, malgré leur aspect spectaculaire qui n'a pas pu échapper aux observateurs. En effet, certains des pierriers ou murgers (nous emploierons indifféremment les deux termes) qui les composent, appelés localement Steinrudeln, ont des dimensions impressionnantes: plus de $10 \mathrm{~m}$ de large et $4 \mathrm{~m}$ de hauteur pour une longueur de plusieurs centaines de mètres. Ces structures échappent également à la mémoire collective villageoise. Les habitants d'Osenbach interrogés répondaient invariablement que les Steinrudeln dataient du $19^{\mathrm{e}}$ siècle «à l'époque où l'on cultivait les pommes de terre sur le Bickenberg ", alors qu'à l'évidence le fort émoussé géochimique des arêtes de très nombreuses pierres gélifractées in situdans les pierriers était incompatible avec une telle datation. L'âge de ces structures, leur origine, les raisons mêmes de l'existence de ce maillage lithique dans une région d'openfield étaient complètement inconnus.

3 Afin de répondre à quelques-unes de ces questions, un mémoire de maîtrise de géographie a été engagé à titre exploratoire sous la direction de D. Schwartz sur le Bickenberg, colline sur laquelle les structures étaient le mieux conservées. Ce travail (Bernardy, 1998) a bénéficié d'une aide logistique et financière de la municipalité d'Osenbach et d'un mécénat d'entreprise, ce qui a permis de procéder à la datation ${ }^{14} \mathrm{C}$ de quelques structures. Il a été réinterprété et complété par des observations inédites (D. Schwartz), tandis qu'un travail comparatif était effectué sur les autres collines du secteur (Vonna, 2003) et que d'autres aspects, concernant notamment les enjeux actuels de la gestion de ces espaces, sont développés dans une thèse en cours (Litaudon, 2004).

4 Ce sont ici les résultats obtenus sur le Bickenberg qui seront développés. Toutefois, quelques données issues des travaux effectués par Litaudon-Jouve et Vonna seront également utilisées pour étayer certains raisonnements.

\section{Localisation et caractéristiques}

5 Le Bickenberg constitue avec le Zinnkoepflé, le Strangenberg et le Bollenberg un ensemble de collines sèches calcaires grossièrement inscrit dans le triangle Rouffach Osenbach - Orschwihr (fig. 1). La sécheresse de ces collines est liée à la conjonction de trois facteurs : un soubassement formé de différents types de calcaires très diaclasés et de ce fait poreux ; la pauvreté de la réserve hydrique des sols, qui sont des sols calcaires peu épais (lithosols, rendzines et sols bruns calcaires); des précipitations faibles (550 à $600 \mathrm{~mm} / \mathrm{an}$ ) liées à la position d'abri sous le vent à l'est des Vosges. Il en résulte une végétation xérophile et thermophile très particulière, comprenant de nombreuses plantes rares et présentant des affinités avec à la fois des groupements méditerranéens et d'Europe Centrale; une bonne partie de ces pelouses est de ce fait protégée. En dehors des zones viticoles, la végétation est principalement constituée d'une pelouse à Bromus erectus. Toutefois, la fruticée, constituée de nombreux arbustes souvent épineux (prunelliers, églantiers, aubépines, cornouillers, viornes...), a tendance à gagner du terrain sur ces pelouses maintenant sous-pâturées (Hoff, 1978; Turlot, 1978; Paul, 1983 ; Jacob, 1984 ; Sell et al., 1998). Si l'origine anthropique des pelouses ne fait aucun 
doute, l'âge de ces formations est totalement inconnu, même si des défrichements néolithiques sont parfois évoqués (Jacob, 1984), tandis que Sell et al. (1998) suggèrent - très prudemment - qu'une partie des pelouses (celles établies sur les lithosols) pourraient être naturelles, puis avoir été agrandies par le pâturage.

Figure 1 : Carte de localisation des collines sèches du secteur de Rouffach.

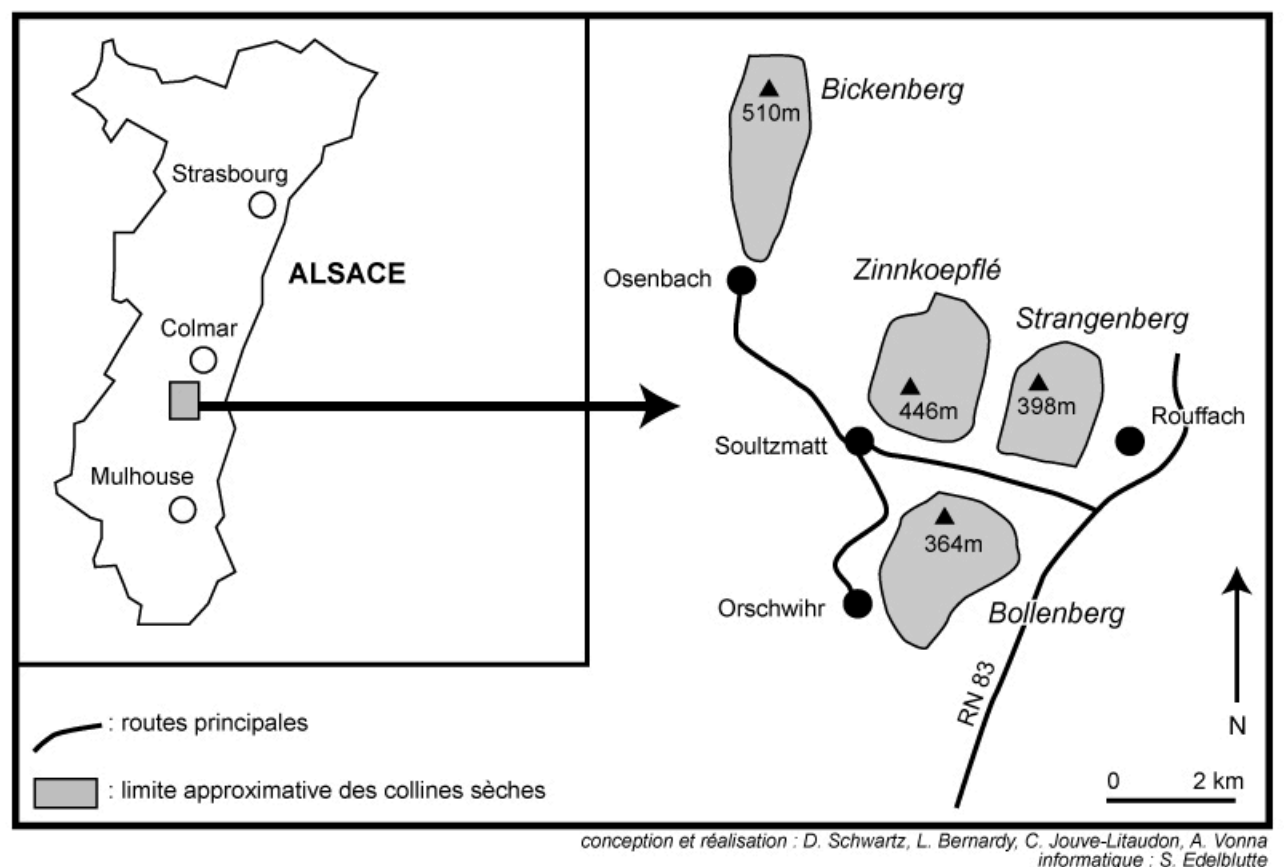

6 Le Bickenberg est la plus occidentale, la plus septentrionale et la plus élevée (450 à 550 $\mathrm{m})$ des collines sèches. Situé au pied même des Hautes-Vosges, les caractères de sécheresse y sont un peu moins accentués que sur les autres secteurs. Le soubassement est ici constitué de calcaires du Muschelkalk (Théobald, 1977). Le Bickenberg constitue un éperon grossièrement orienté N-S (Tricart et Usselmann, 1967). La partie méridionale forme une surface tabulaire à pente douce inclinée vers le sud. Les versants, bien marqués, sont dissymétriques; le versant ouest est bien plus raide que le versant est. Cette zone a connu une évolution notable de son couvert végétal depuis les années 1950-60. Pour schématiser, à cette époque le versant ouest était occupé par une pelouse de type Mesobrometum, la zone tabulaire était cultivée et les versants sud et est étaient couvert de vergers et de vignes. Actuellement, le versant ouest est recouvert d'une chênaie de reconquête, le plateau est en totalité en prairies de fauche et en pâturages, le versant est en grande partie occupé par une fruticée, la vigne ayant complètement disparu. Quant à la partie septentrionale du Bickenberg, elle s'élève en pente relativement forte vers les Vosges et est couverte de forêt. Les Steinrudeln se développent exclusivement sur la partie tabulaire du Bickenberg. Ils sont soulignés par un ourlet forestier.

\section{Méthodes d'étude}

Plusieurs approches croisées ont été développées pour répondre aux objectifs fixés. 
8 En premier lieu, il s'agissait de pouvoir cerner la structure d'ensemble du dispositif, et d'en préciser les caractéristiques morphologiques. A cet effet, une cartographie quasiexhaustive des pierriers a été effectuée à l'aide de relevés au décamètre, reportés sur les plans cadastraux en se servant du bornage et de calages à l'aide des photographies aériennes. Une carte d'ensemble a été ensuite reconstituée en réduisant l'échelle des relevés. La stratigraphie des pierriers a été étudiée sur plusieurs coupes liées à une exploitation ponctuelle de ces structures en tant que matériau d'empierrement (pour le drainage de constructions individuelles). Ces approches de terrain ont été complétées par l'établissement d'une typologie des pierriers fondée sur leur morphologie et la présence ou non d'un appareillage, par une évaluation, forcément grossière, du volume représenté par les murgers et par une étude des distances entre structures parallèles pour en étudier la récurrence. L'appareillage est en fait constitué par une base de muret en pierres sèches évitant que le pierrier n'empiète sur les terres situées à proximité immédiate.

Figure 2 : Localisation des prélèvements $14 \mathrm{C}$ sous les pierriers $\operatorname{Pr} 1$ et $\operatorname{Pr} 2$ (repérés sur la figure 3 ).

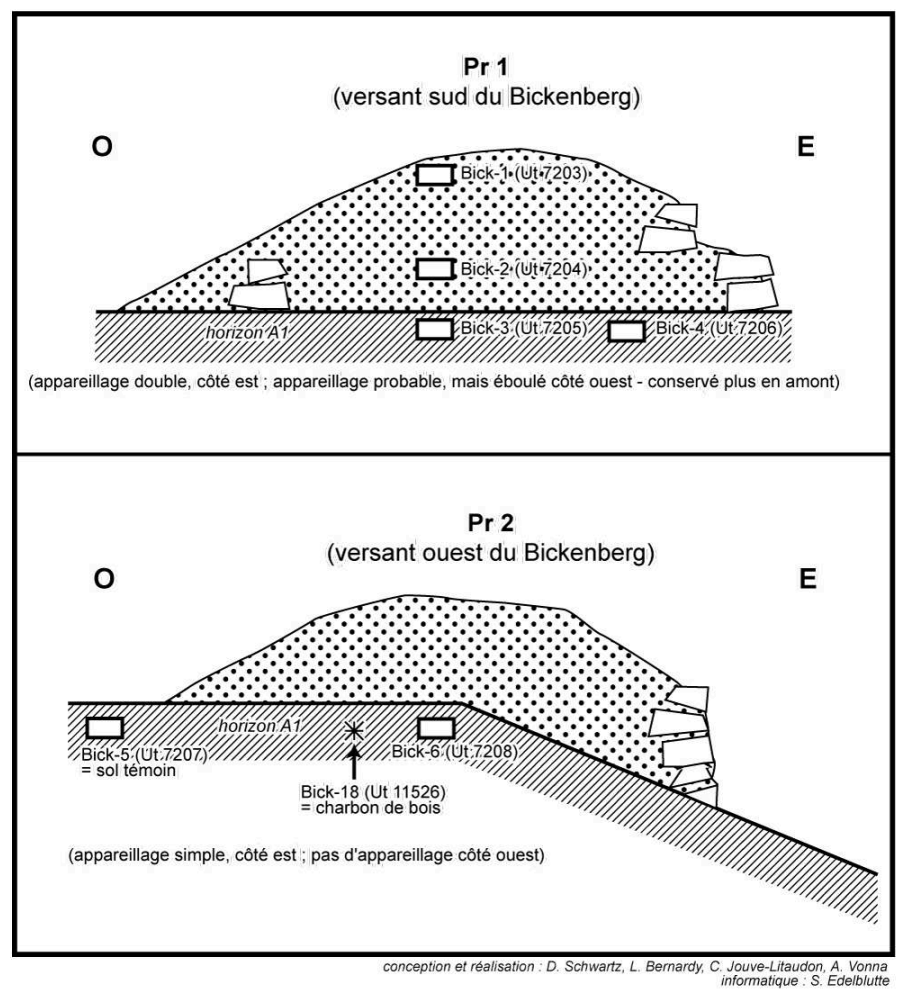

Différents types de prélèvements ont été effectués dans l'objectif de dater au ${ }^{14} \mathrm{C}$ les pierriers (fig. 2). Deux pierriers ( $\operatorname{Pr} 1$ et $\operatorname{Pr} 2$ ) ont été excavés sur deux versants différents (fig. 3) afin de prélever l'horizon humifère A1 du paléosol conservé sous les structures. Nous avons préféré échantillonner ainsi plutôt que de prélever dans les zones exploitées comme matériau d'empierrement afin de limiter les risques de perturbations, cela malgré les difficultés techniques du creusement des fosses dans ce matériau instable. La mesure d'âge ${ }^{14} \mathrm{C}$ des matières organiques d'un paléosol enfoui donne en principe l'âge de l'enfouissement, augmenté de l'âge moyen qu'avaient les matières organiques au moment de leur recouvrement par le matériau plus récent (Guillet, 1979). Afin de déterminer l'âge de l'enfouissement, il est donc indispensable de connaître ce dernier paramètre, qui représente en fait le temps moyen de résidence 
(TMR) des matières organiques dans l'horizon daté. En principe, ce TMR est peu élevé dans les horizons de surface. Nous avons toutefois prélevé et daté un humus actuel à proximité immédiate d'une des deux fosses ouvertes pour en avoir une mesure directe. Par ailleurs, la protection assurée contre l'activité biologique par l'enfouissement n'est que partielle dans ce type de matériau dont les volumes de vide interstitiels sont très importants. Il est tout à fait possible que les matières organiques soient partiellement renouvelées par l'activité faunistique du sol, ce qui provoque un rajeunissement des échantillons mesurés. Aussi est-il prudent de considérer l'âge obtenu par les datations ${ }^{14} \mathrm{C}$ non pas comme l'âge réel de l'enfouissement, mais comme son âge minimal. La matière organique interstitielle des pierriers a également été datée. Nous avons par ailleurs extrait des microcharbons de bois de l'horizon A1 du paléosol conservé sous $\mathrm{Pr}$ 1. Un de ces charbons a été daté. L'enfouissement des charbons précède en principe l'érection des murgers. En conséquence, la datation donne ici l'âge maximal des pierriers. Les deux types de mesures d'âge, sur matière organique et sur charbon, permettent donc d'encadrer l'époque d'érection des murgers. Enfin, la construction en 2002 d'une maison rue Saint-Marc, au pied du versant S.E. du Bickenberg nous a fourni l'opportunité d'étudier une très belle coupe stratigraphique dans laquelle un petit lit de charbons situé à la base de colluvions agricoles a également été prélevé et daté. Au total, ce sont donc 8 datations qui ont été faites, auxquelles s'ajoutent à titre de comparaison deux datations de charbons prélevés sous un pierrier du Strangenberg dans le cadre de la thèse de C. Litaudon-Jouve (fig. 2, tabl. 1).

Tableau I : Résultats des datations 14C

\begin{tabular}{|c|c|c|c|c|c|c|}
\hline$N^{\circ} E c h$ & $N^{\circ} l a b o$ & Nature & Age $14 \mathrm{C}$ brut (BF) & Age calibré (BP) & Age calibré (AD) & $T M R^{*}$ \\
\hline Pr1/Bick-1 & Ut-7203 & matière organique & $-1731 \pm 37$ & sans objet & - & $26 \pm 1,5$ \\
\hline Pr1/Bick-2 & Ut-7204 & matière organique & $-571 \pm 39$ & sans objet & - & $128 \pm 6$ \\
\hline Pr1/Bick-3 & Ut-7205 & matière organique & $396 \pm 42$ & sans objet & - & $658 \pm 40$ \\
\hline $\operatorname{Pr} 1 /$ Bick-4 & Ut-7206 & matière organique & $175 \pm 38$ & sans objet & - & $470 \pm 25$ \\
\hline Pr1/Bick-5 & Ut-7207 & matière organique & $1098 \pm 34$ & sans objet & - & $62 \pm 2$ \\
\hline Pr1/Bick-6 & Ut-7208 & matière organique & $429 \pm 45$ & sans objet & - & $690 \pm 40$ \\
\hline $\begin{array}{l}\text { Osenbach- } \\
\text { St-Marc }\end{array}$ & Ut-11525 & charbon & $1250 \pm 50$ & $\begin{array}{c}261-1168,1160-1136 \\
1107-1092\end{array}$ & $\begin{array}{c}689-782,790-814 \\
843-858\end{array}$ & - \\
\hline $\begin{array}{l}\text { Osenbach- } \\
\text { St-Marc }\end{array}$ & Ut-11526 & charbon & $369 \pm 29$ & $484-430,361-326$ & $1466-1520,1589-1624$ & - \\
\hline Stran-I1 & Ut-11520 & charbon & $1000 \pm 110$ & $\begin{array}{c}\text { 1051-1030, 992-787 } \\
777-770\end{array}$ & $\begin{array}{c}99-920,958-1163 \\
1173-1180\end{array}$ & - \\
\hline Stran-IB & Ut-11521 & charbon & $1130 \pm 50$ & $\begin{array}{c}166-1162,1118-1113 \\
1074-96\end{array}$ & $\begin{array}{c}\text { 784-788, 832-837 } \\
876-984\end{array}$ & - \\
\hline
\end{tabular}

10 Ces approches ont été complétées par l'étude des quelques archives historiques disponibles sur le secteur : cartes, cadastre napoléonien, minutes de procès, terriers.

\section{Les résultats et leur interprétation}

\section{Cartographie des pierriers}

La cartographie des pierriers révèle la grande cohérence générale du dispositif du Bickenberg (fig. 3), qui est sans conteste celui dont la structure d'ensemble est la mieux conservée. En effet, si les structures étudiées par Vonna (2003) sur le Strangenberg 
(photo 1) et le Zinnkoepflé apparaissent plus complexes, et sans doute mieux conservées par endroit, il n'est pas possible de relier entre eux les différents secteurs cartographiés sur chaque colline. L'élément de base du dispositif est un grand murger périphérique qui ceinture le Bickenberg à la rupture de pente entre le plateau et les versants quasiment en continu sur 1,5 $\mathrm{km}$ de long. Par endroit, ce murger fait plus de $10 \mathrm{~m}$ de large et $4 \mathrm{~m}$ de haut en son centre.

Figure 3 : Cartographie d'ensemble des Steinrudeln du Bickenberg.

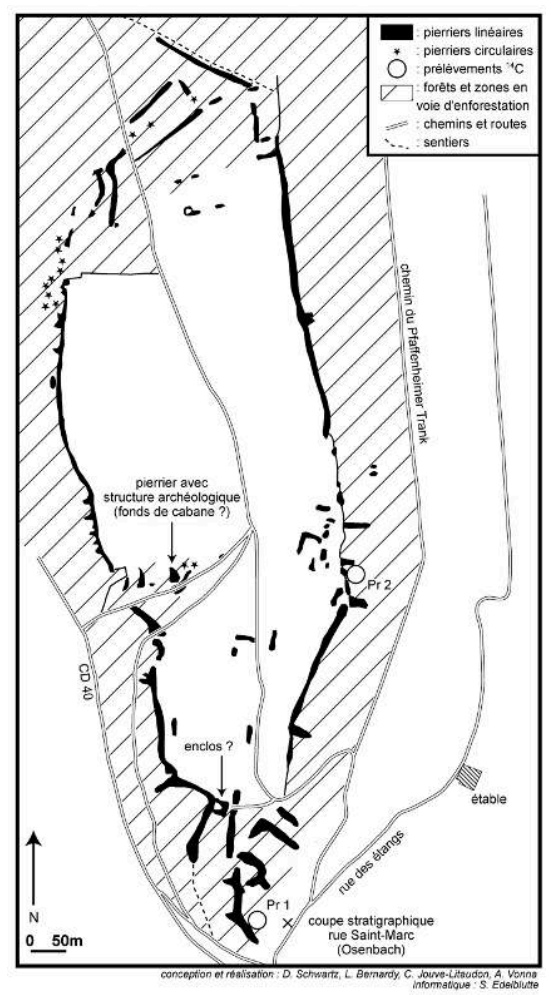

Complété et modifié de Bernardy (1998) 
Photo 1 : Un aspect du parcellaire réticulé délimité par les Steinrudeln sur le flanc ouest du Strangenberg (Westhalten).

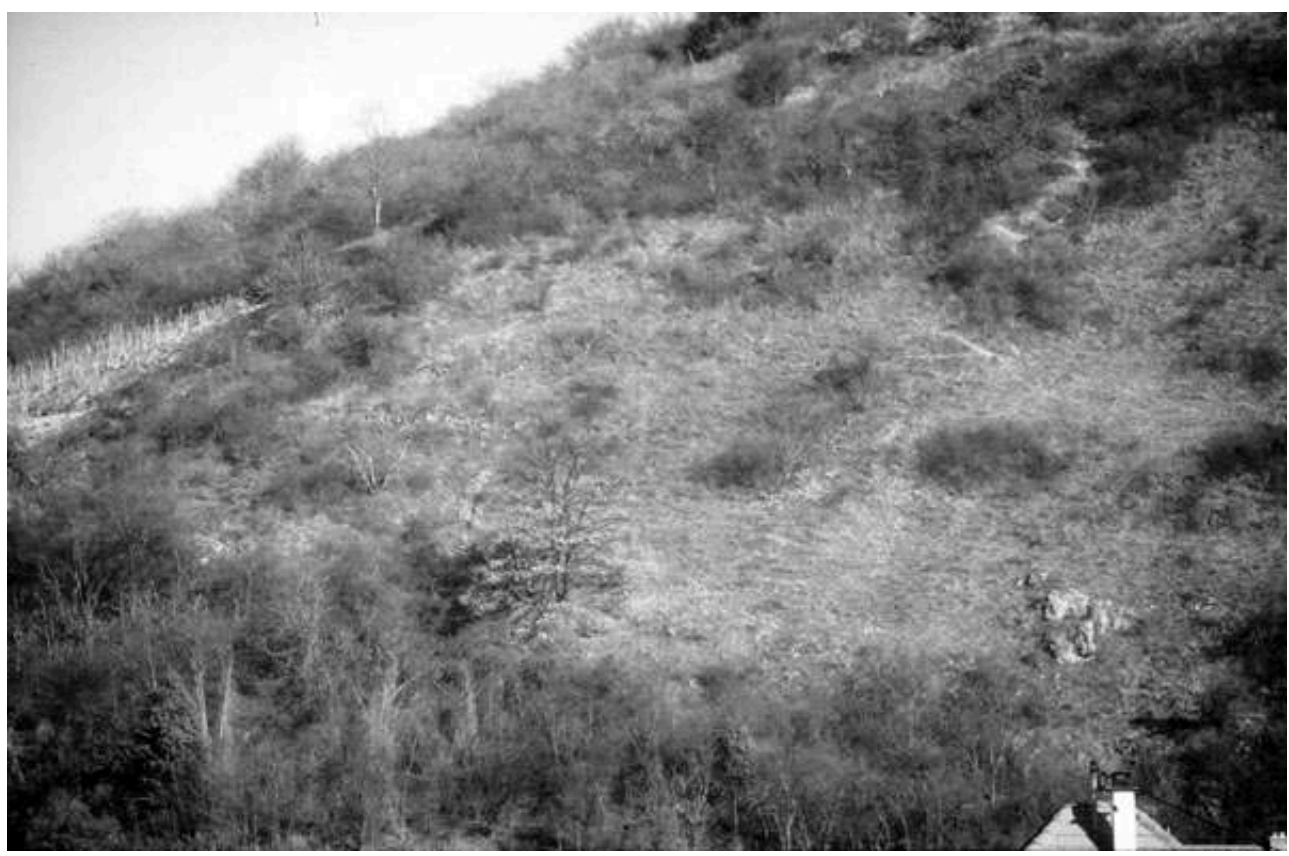

Une partie des pierriers est visible ; l'alignement des bosquets souligne la présence de ceux qui sont masqués par la végétation

Dominique Schwartz

Cette structure, soulignant le relief particulier du Bickenberg, n'est pas sans évoquer les éperons barrés protohistoriques. Il s'agit à notre sens d'une simple convergence de forme. Aucun vestige archéologique n'a jamais été découvert sur le Bickenberg, même lorsque les terrains étaient labourés. L'absence totale de structure d'habitat, de découverte d'objets tels que des tessons de céramique, des éclats de silex, ou à plus forte raisons d'artefacts plus élaborés (fusaïoles, meules, molettes,...), ou encore de simples débris osseux permet d'écarter l'hypothèse de ruines d'une forteresse et/ou d'un habitat protohistoriques ou médiévaux.

Sur le versant est du Bickenberg, le côté d'un pierrier principal donnant sur la pente est soigneusement appareillé sur près de $1 \mathrm{~m}$ de haut, ce qui n'est pas le cas sur le versant ouest plus raide. A l'intérieur de cette structure, les murgers sont plus rares, à l'exception de deux secteurs, l'un tout au nord, et l'autre dans la partie est. Mais il semblerait qu'une partie des murgers aient disparu, utilisés pour l'empierrement du C.D. 40 qui part d'Osenbach vers Munster (renseignement oral). De fait, sur le terrain, plusieurs structures actuellement séparées les unes des autres semblent bien avoir été initialement en continuité. De nombreux murgers radiaux viennent s'appuyer sur la structure principale sur les versants est et sud, où ils sont particulièrement impressionnants (photo 2). La majorité d'entre eux sont appareillés sur leurs deux faces, certains d'entre eux présentant même deux niveaux de murets décalés. Le versant ouest est bien différent: les murgers radiaux sont bien plus courts, non appareillés. Apparaissent également (au N.O.) des amas subcirculaires répartis de façon assez aléatoire. La différence entre les deux versants pourrait être liée à une différence dans l'utilisation du milieu. Le fait que l'appareillage soit particulièrement soigné le long des versants est et sud indique qu'on a scrupuleusement veillé à ne pas trop empiéter sur les terres dans cette zone. Ceci suggère que, contrairement à l'autre 
versant plus raide, ces terres étaient également destinées à un usage agricole au moment de l'érection des Steinrudeln.

Photo 2 : Aspect général d'un pierrier linéaire radial situé sur le flan sud du Bickenberg (à l'est du pierrier $\operatorname{Pr} 1$ de la figure 3)

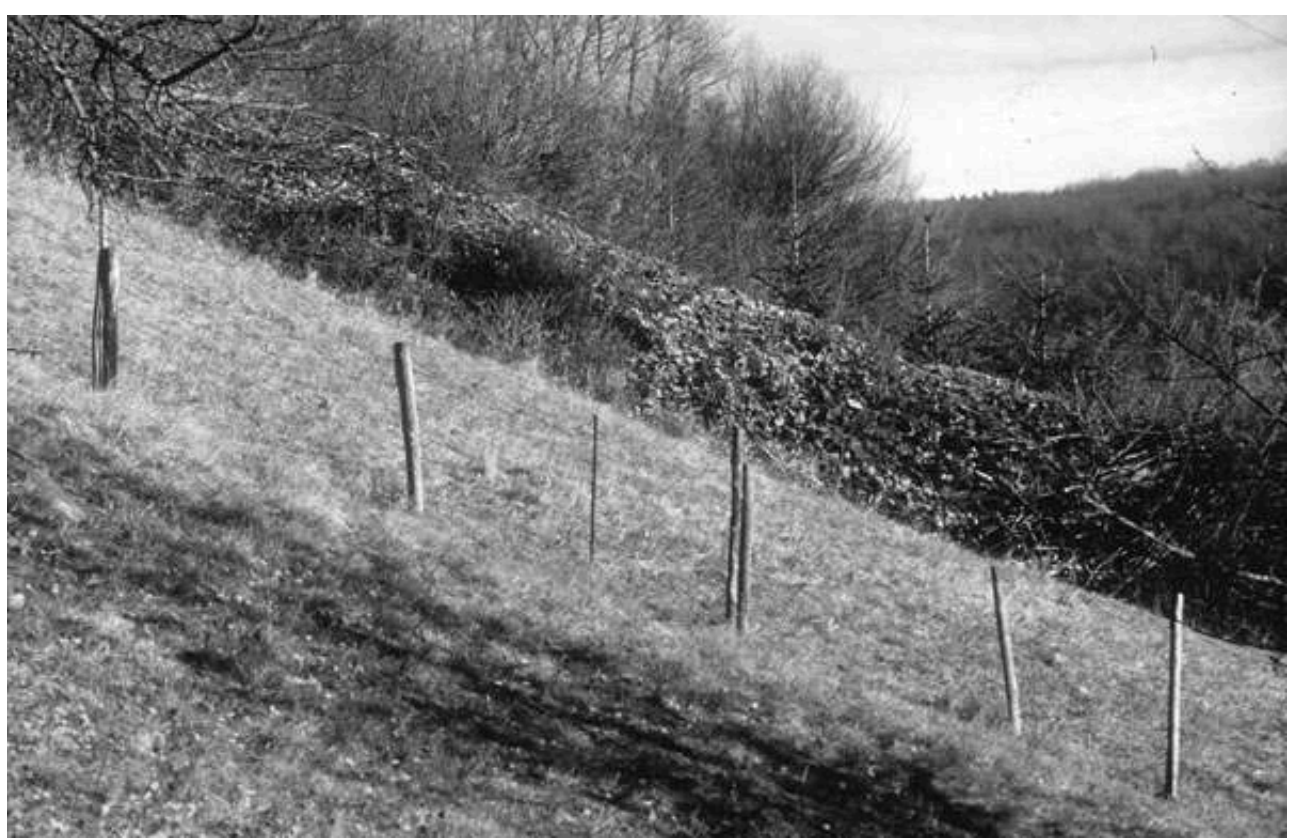

Dominique Schwartz

Cette cartographie a également mis en évidence trois structures particulières, qui méritent d'être relevées ici.

- La première est un pierrier ceinturant une parcelle carrée d'environ 15-20 m de côté extérieur et de 10-12 m de côté intérieur, ce qui exclut que cette parcelle n'ait jamais pu être labourée par un attelage. Peut-être s'agit-il d'un enclos (fig. 3).

16 - La deuxième est une curieuse excavation carrée d'environ 2 區 $2 \mathrm{~m}$ dans un pierrier massif d'environ 15 蠋 $20 \mathrm{~m}$ de dimension (fig. 3). Cette fosse fait actuellement environ 1 $\mathrm{m}$ de profondeur. Elle est soigneusement appareillée par un muret en pierres sèches sur sa face interne, mais pas du tout à l'extérieur où elle se fond dans le pierrier. Ceci démontre que cette structure est rigoureusement contemporaine de l'érection du pierrier. Peut-être s'agit-il d'un fond de cabane destiné à abriter temporairement des travailleurs participant aux travaux d'épierrement. Quelques structures analogues ont été observées par Vonna (2003). Elles mériteraient de faire l'objet d'une fouille archéologique.

17 - La dernière est un assez grand bâtiment ( 8 䔾 $5 \mathrm{~m}$ ), constitué de deux pièces au sol et effondré. Situé à environ $50 \mathrm{~m}$ à l'est de la précédente structure, il est construit en pierres sèches calcaires du Muschelkalk local. Ce type de construction est rare en Alsace. Il n'est pas relié directement à un pierrier, mais son appartenance à ce système semble probable. 


\section{Structure interne et stratigraphie des pierriers}

L'observation en coupe des pierriers montre qu'ils sont composés de blocs de tailles variées. Les plus gros de ces blocs dépassent $60 \mathrm{~kg}$ et sont constitués par des fragments complets de bancs calcaires du Muschelkalk. Les plus petits n'ont que 2 ou 3 centimètres d'arête. Ces derniers ne résultent pas directement de l'épierrement. Non seulement il aurait été illusoire de vouloir extraire du sol les cailloux de cette taille, mais les préceptes agricoles anciens, dont Olivier de Serres se fait encore l'écho en 1600 , recommandaient de laisser les pierres de cette taille dans les sols lourds. L'aspect de ces pierres, et plus particulièrement l'allure fraîche d'un certain nombre d'arêtes montre qu'il s'agit de blocs plus importants gélifractés. Ils sont d'ailleurs bien plus abondants dans la partie supérieure des Steinrudeln, et il nous a été possible à maintes reprises de reconstituer le bloc d'origine. Les arêtes de nombreuses pierres gélifractées ont subi un émoussé géochimique après leur empilement dans les Steinrudeln. L'intensité de ces phénomènes de dissolution suggère un âge ancien de ces structures. Au sein même des pierriers, les blocs n'ont aucune orientation privilégiée. Ce point a pu être aisément vérifié car de nombreux blocs proviennent de bancs calcaires normalement revêtus à leur face inférieure, en position stratigraphique dans des coupes géologiques, de calcite de reprécipitation formant des concrétions de type «chou-fleur». Dans les pierriers, l'orientation de ces concrétions est totalement aléatoire.

Par ailleurs, l'empilement des blocs dans les Steinrudeln se fait en général sans matrice fine interstitielle, au moins dans les niveaux les plus profonds. Les vides représentent une partie importante du volume total des pierriers. Cependant, dans les nombreux Steinrudeln recouverts d'arbres, la décomposition des litières provoque un début de pédogenèse, et les vides sont plus ou moins comblés sur quelques dizaines de centimètres d'épaisseur par une matrice fine interstitielle, riche en matière organique et de teinte sombre. Cette matrice organo-minérale est formée de petits agrégats d'origine biologique (pellets d'enchytréides). Dans certains cas elle est suffisamment développée pour former un véritable anthroposol, de type ranker d'éboulis ou sol humo-calcique.

D'un point de vue stratigraphique, les pierriers constituent en général un ensemble très homogène. Les discontinuités décrites ci-dessus, qu'il s'agisse de la gélifraction provoquant une plus grande abondance de pierres de petite taille dans la partie supérieure des Steinrudeln, ou de la présence d'une matrice fine pédologique n'ont en effet pas de valeur stratigraphique. Or, en dehors des variations liées à ces deux types de phénomènes, aucune discontinuité n'a jamais pu être observée dans les coupes relevées. Tout au plus, dans certains, est-il possible de distinguer deux ensembles différents :

- une couche supérieure, d'épaisseur inférieure à $30 \mathrm{~cm}$, qui contient de nombreux vestiges

récents (débris de bouteilles, de vaisselle, fragments d'ustensiles de cuisine émaillés et

d'outils métalliques,...);

- une couche inférieure, souvent plurimétrique, ne contenant aucun vestige d'origine archéologique, ni discontinuité lithologique.

21 En conclusion, les caractéristiques générales des pierriers permettent de faire l'hypothèse qu'ils ont été édifiés d'une traite, sans doute assez rapidement. Seule leur partie supérieure a été localement remaniée par les activités agricoles des dernières 
décennies (et peut-être du XIX ${ }^{\mathrm{e}}$ siècle), celles dont se souviennent les anciens du village qui ont vu leurs parents, leurs grands-parents y déverser des seaux de pierres, et qui ont eux-mêmes bien souvent participé à ce travail. Mais en dessous de ce niveau, la grosse masse des pierriers correspond à une seule phase d'édification. Dans le cas contraire, c'est-à-dire avec différentes phases de formation séparées par des intervalles de temps longs, on devrait identifier plusieurs niveaux stratigraphiques, contenant pour certains des restes archéologiques, ou bien des sol enterrés du même type que ceux qui se développent actuellement sur les pierriers (rankers d'éboulis et sols humocalciques).

\section{Coupe stratigraphique de la rue Saint-Marc à Osenbach}

La construction, courant 2002 d'une maison rue Saint-Marc, au pied du versant sud du Bickenberg nous a permis d'observer une très belle coupe stratigraphique, haute d'environ 3,50 m (fig. 4). La base de la coupe est constituée sur environ 1,90 m observables d'une formation de pente, constituée de blocs décimétriques de calcaire du Muschelkalk. Ces blocs sont enrobés dans une matrice fine, argilo-calcaire, dans laquelle on peut également observer des précipitations de carbonates secondaires. Ils sont d'origine locale, mais contiennent très localement quelques blocs de grès. Ceux-ci ne peuvent provenir que du versant opposé du vallon du Pfaffenheimer Trank, constitué de grès à meules du Buntsandstein moyen. Ils traduisent une divagation épisodique du ruisseau du Pfaffenheimer Trank, dont le tracé d'origine tectonique sépare, le long d'une faille, la rive droite calcaire de la rive gauche gréseuse. Cette formation est entaillée à deux endroits par un chenal ravinant, entaillant le versant dans le sens de sa plus grande pente, et comblé par un matériau limoneux calcaire qui semble être du loess remanié. L'ensemble de cette formation est d'origine périglaciaire, sans précision sur son âge réel. Les formations attribuées au Würm sont très abondantes dans le secteur (Tricart et Usselmann, 1967). Cette formation est recouverte par des colluvions sur une épaisseur de 1,65 m. Leur grande épaisseur contraste fortement avec celle des sols du versant (rendzines et sols bruns calcaires épais au plus de $50 \mathrm{~cm}$ ). Ces colluvions sont relativement homogènes sur toute leur épaisseur, à l'exception de l'horizon humifère qui se développe sur les $20 \mathrm{~cm}$ supérieurs. Elles sont constituées pour l'essentiel d'une matrice fine argilo-limoneuse, de teinte brune, carbonatée, contenant d'abondants fragments de calcaires du Muschelkalk de taille centimétrique. Des charbons de bois dispersés dans la masse peuvent être observés sur toute l'épaisseur du profil. Ils sont plus abondants tout à la base des colluvions. Ils ont été prélevés systématiquement sur toute l'épaisseur des colluvions, mais pour l'heure seul le niveau prélevé tout à la base des colluvions a été daté. Le contact entre les deux formations constitue une discontinuité stratigraphique majeure. Les colluvions témoignent d'une déstabilisation du versant postérieure aux temps glaciaires. Ils sont sans conteste d'origine agricole et datent donc les débuts de la mise en culture du Bickenberg. 
Figure 4 : Vue schématique de la coupe stratigraphique de la rue Saint-Marc.

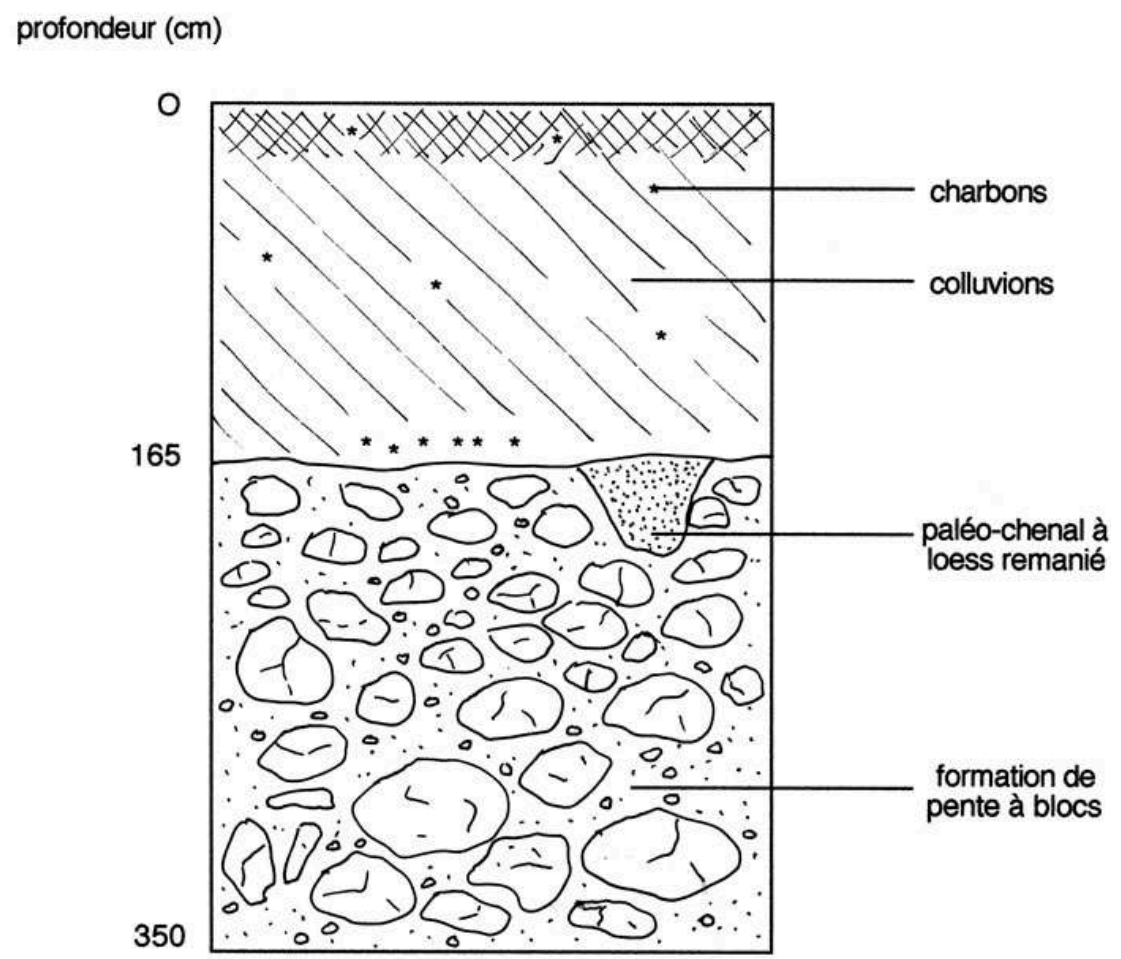

\section{Récurrences de distances et métrique utilisée}

Un certain nombre de distances récurrentes reviennent régulièrement dans le parcellaire. Ainsi, sur le flanc est du Bickenberg, 4 murgers successifs sont séparés par des distances de 54, 54 et $216 \mathrm{~m}$ (soit 4 fois $54 \mathrm{~m}$ ) (fig. 3). Cette récurrence de $54 \mathrm{~m}$, de diviseurs $(18,27,36)$ et plus rarement de multiples $(72,216)$ se retrouve à différents endroits sur le Bickenberg. Elle se retrouve également dans le cadastre actuel. Ainsi, le long du chemin du «Pfaffenheimer Trank », qui longe le versant Est du Bickenberg, on observe 9 fois d'affilée cette distance de $54 \mathrm{~m}$ comme limite de parcelles. Pour cette raison, une première hypothèse a été proposée à titre exploratoire par Monsieur $\mathrm{J}$. Ehrart, Président de la Société d'Histoire de Rouffach : l'utilisation comme unité de mesure de la coudée rouffachoise, dont l'étalon de $54,1 \mathrm{~cm}$ est gravé sur la façade de l'église de Rouffach (Bernardy, 1998). L'unité de mesure de distance aurait dans ce cas été la centaine de coudées. Cette hypothèse a priori séduisante ne résiste cependant pas à l'analyse : la coudée n'est pas utilisée comme unité de mesure agraire, les métriques utilisées par le passé ne faisaient jamais appel au système décimal, mais au système vicésimal (multiples de vingt), et des distances telles que 18 ou $36 \mathrm{~m}$ ne sont même pas des multiples entiers de la coudée.

Dans son étude du Strangenberg et du Zinnkoepflé, Vonna (2003) a repris ce point sur une base statistique, grâce à l'existence d'un assez grand nombre de pierriers parallèles. Aux erreurs de mesure près, et en tenant compte de l'imprécision sur la distance réelle à considérer (l'axe des pierriers ? la distance entre deux bords ?), il semble que les distances les plus représentées soient en fait des distances de 17-19 m ou 34-38 m, soit en moyenne 18 et $36 \mathrm{~m}$. On retrouve également de tels écartements sur le 
Bickenberg, et on remarquera enfin que $54 \mathrm{~m}$ est un multiple de $18 \mathrm{~m}$. Ceci plaide en faveur de l'emploi du pied, normalisé par Charlemagne $(30,48 \mathrm{~cm})$. Les distances de 18, 36,54 et 216 m correspondant respectivement à $60,120,180$ et 720 pieds, ce qui cadre bien avec un système vicésimal.

\section{Analyse des archives historiques}

Le dépouillement des archives historiques n'a pas apporté de résultats probants pour les périodes les plus reculées.

La plus ancienne référence à une activité agricole sur le Bickenberg se trouve dans un dossier des archives du couvent Saint-Marc (1768: AD68, 2H, carton 3, n 14). Ce dossier contient les «Titres, actes et pièces d'une procédure au sujet du droit d'usage prétendu par le Prieuré de Saint-Marc dans la forêt dite Bickenberg, appartenant à la commune de Pfaffenheim, de 1735 à 1768 ». On y lit que le «30 juillet 1735 à la requête des Prevost Bourgmestre jurés habitans et communauté de Pfaffenheim qui font élection de domicile en la [illisible] du sieur Kneist prévot en dit lieu, soit sommé et interpellé le père Blaise Kesser, prevost de Saint-Marc, de donner communication et copie fidèle de la lettre et titre en entier de l'année 1258 en vertu duquel il prétend avoir les droits de pâturage et de glandage dans le ban et forêt du dit Pfaffenheim pour les requéreurs et pouvant répondre... ». Cette pièce pourrait indiquer qu'en 1258 co-existaient au Bickenberg des forêts de chêne, dont les glands servaient à nourrir les porcs, et des pâturages. Un paysage finalement pas très différent de l'actuel, assez semblable à celui représenté par Jean Colombe en 1485 pour le mois de Novembre dans une célèbre enluminure des Très riches heures du Duc de Berry (Dufournet, 1995). La prudence doit toutefois être de mise, le droit de pâture ne s'exerçant pas forcément dans des pâturages au sens moderne du terme, mais aussi en forêt. Le titre du dossier suggère d'ailleurs qu'il s'agit bien d'un droit de pâturage en forêt : tout le Bickenberg était-il couvert de forêt à cette date, ou bien seulement la partie qui l'est de nos jours? Rien ne permet de répondre précisément à ces interrogations.

Les deux autres documents retrouvés aux Archives Départementales du Haut-Rhin (sér. 13H) ne permettent pas plus de répondre à ces questions. Le premier d'entre eux est un extrait des archives de l'abbaye de Marbach, daté de 1356. Il mentionne, entre autres, une taxe due par le tenancier Dietrich Schappeler, fils de Dietrich et de Catherine, pour un verger situé au Bickenberg: item, duo pomaria unum in Tieffenwege et unum in Bickenberg est (De même, deux vergers, l'un situé le long du chemin creux, l'autre au Bickenberg...). Le terme de pomaria est trompeur : on pense à des pommiers, mais il désigne n'importe quel type de verger. Ce document est pour l'instant le plus ancien dans lequel apparaît directement le nom du Bickenberg. Le deuxième est l'Urbaire de Marbach, daté de 1488: "Item, Martin Bryser, 7 schilling von einem Matten ist 1 juch im Rystal neben Claven Hungerstein dem jungen ander sit neben der Sloyffen die im Byckenberg get und stosset oben an das Odestophelin " (De même, [pour] Martin Bryser, 7 schillings [de taxes] pour un pré d'un arpent (soit 40 ares) situé au Rystal, entre [celui de] Claven Hungerstein le jeune, et de l'autre côté du Sloyffen montant au Bickenberg et arrivant à l'Odestophelin). Le terme Odestophelin est resté intraduisible, sa signification nous échappant totalement. Les "Schlauffa" étant plutôt des sentiers de débardage, il semblerait que le Bickenberg était à cette époque recouvert, au moins partiellement, de forêts. 
La lecture de ces archives ne permet pas réellement de conclure quant à l'importance de l'utilisation agricole du Bickenberg, ni de celle de la couverture forestière. Elle ne nous apporte en particulier aucun renseignement sur l'édification des murgers. En effet, aucun des usages avérés ou supposés (l'arboriculture fruitière, le pâturage sur prairie) ne nécessitent ou justifient un épierrement de cette ampleur. On a parfois avancé le fait que l'épierrement permettait de délimiter des parcelles de pacage. Outre le fait que ces limites sont peut compatible avec un pâturage itinérant de règle pour les ovins jusqu'il y a peu, cette explication parait réductrice et peu en accord avec les faits. Le travail demandé pour l'élaboration des pierriers est sans commune mesure avec le résultat demandé, de simples haies végétales tressées en coudrier faisant parfaitement l'usage, attesté dans de nombreux documents médiévaux. De plus, même en tenant compte qu'ils ont pu partiellement s'ébouler, la plupart des pierriers ne sont pas assez abrupts ou élevés pour retenir efficacement des animaux, quels qu'ils soient. Il aurait fallu qu'ils soient rehaussés par une haie végétale. Cela est possible, mais quel est l'intérêt alors de les élaborer, si les haies de coudriers tressés suffisent? Comment expliquer la présence des colluvions agricoles si le seul usage de ces terrains a été la pâture ? La taille si restreinte de nombreuses parcelles?

L'occupation des sols est en revanche bien connue à compter de 1760. Un plan de finage du baillage de Soultzmatt distinguait à cette date 5 types d'occupation sur la zone : les terres cultivées, les prairies de fauche, les pâturages, les vignes et les forêts. Ce plan montre clairement que toute la partie tabulaire du Bickenberg était à cette époque en pâture, alors que le vallon du Pfaffenheimer Trank qui le jouxte au pied du versant est était pour sa part occupé par des cultures et des prairies de fauche. A Soultzmatt, le Zinnkoepflé est également cartographié en pâturage. En revanche, en 1817, le plan d'ensemble du cadastre napoléonien montre que quelques parcelles du versant est du Bickenberg étaient couvertes de vigne, les autres restant en pâtures. C'est donc entre 1760 et 1817 que la vigne a été plantée le long du Bickenberg. Outre le fait qu'un vignoble n'est pas forcément épierré, cette date tardive permet d'écarter la viticulture comme origine possible des Steinrudeln. Les constructions liées à la viticulture se distinguent d'ailleurs nettement des pierriers, par leur structure (des murs et murets en pierre sèche, soigneusement montés et non pas des amas plus ou moins organisés), leur objectif (soutènement et création de terrasses et non épierrement), la nature du matériau (grès vosgien, matériau "noble » apporté depuis des carrières situées sur une colline proche et non calcaire local du Muschelkalk, trop gélif). Lorsque des connexions entre les deux systèmes peuvent être observées, il semble bien que les murets du vignoble viennent s'appuyer sur les Steinrudeln, et non l'inverse. Une autre observation vient étayer ce point. L'écroulement d'un mur de terrasse viticole au pied du versant sud du Bickenberg a permis d'observer le matériau ayant servi à constituer le drainage, large de plus d'un mètre, sur près de $3 \mathrm{~m}$ de hauteur à l'arrière du mur de grès. $\mathrm{Ce}$ matériau est constitué de fragments calcaires d'assez petite taille qui semblent bien avoir été prélevées dans un Steinrudeln situé à proximité immédiate. Ce réemploi tend également à renforcer l'idée de l'antériorité des pierriers par rapport au développement de la viticulture. 


\section{Les datations ${ }^{14} \mathrm{C}$}

30 Les datations ${ }^{14} \mathrm{C}$ discutées ici sont reportées sur le tableau I. Les interprétations doivent tenir compte des spécificités du matériau daté.

31 - La mesure d'âge effectuée sur les matières organiques de l'horizon A1 du sol témoin donne un âge de $-1098 \pm 34$ BP (Pr 2). Cet âge moderne traduit une forte contamination par le ${ }^{14} \mathrm{C}$ résultant des explosions atomiques atmosphériques des années 1945-1970. Les méthodes de calibration classiques, destinées à corriger les âges sur des matériaux biologiques fossilisés (charbons, os, bois,...) ne sont pas applicables ici. Le temps moyen de résidence des matières organiques de cet horizon a été calculé par B. Guillet (Laboratoire de Géochimie organique, CNRS Orléans) en reprenant les données de base (année de prélèvement, activité ${ }^{14} \mathrm{C}$, valeur $\mathrm{du} \mathrm{D}^{14} \mathrm{C}$ ), selon les principes exposés par Balesdent et Guillet (1982). Le temps moyen de résidence ainsi calculé est de $62 \pm 2$ ans.

$32-$ les âges ${ }^{14} \mathrm{C}$ des matières organiques des paléosols conservés sous les pierriers sont respectivement de $396 \pm 42,175 \pm 38$ (Pr1) et $429 \pm 45$ (Pr2) BP. Comme pour l'échantillon précédent, il est impossible de calibrer ces âges pour les exprimer en années calendaires. En revanche, le temps moyen de résidence calculé selon la méthode de Balesdent et Guillet est respectivement de $658 \pm 40,470 \pm 25$ (Pr 1) et $690 \pm 40$ (Pr 2) ans.

33 - les âges des matières organiques interstitielles prélevées dans le murger Pr 1 sont de $-1731 \pm 37$ BP pour celles situées dans la partie supérieure du pierrier, et de $-571 \pm 39$ $\mathrm{BP}$ pour celles situées en profondeur. Ces âges très modernes traduisent, comme pour le sol témoin, une forte contamination par le ${ }^{14} \mathrm{C}$ des bombes atomiques. Ceci démontre qu'une forte activité biologique règne au sein même des pierriers. Recalculés selon la même méthode que précédemment, les temps moyens de résidence sont de l'ordre de $26 \pm 1,5$ et $128 \pm 6$ ans.

34 - Le charbon prélevé sous le pierrier Pr 2 a pour sa part été daté de $369 \pm 29 \mathrm{BP}$, soit un âge calibré de 484-430 ou 361-326 BP. Suivant les principes que nous avons exposés dans les méthodes, ceci signifierait que les pierriers ont au plus 484 ans. Ce résultat est en contradiction totale avec les âges des matières organiques des paléosols. Il ne peut s'expliquer que par une introduction tardive du charbon sous le pierrier. Compte tenu de sa très petite taille (entre 0,4 et $1 \mathrm{~mm}$ de diamètre), il est tout a fait possible qu'il ait été transporté par voie biologique, dans le tube digestif d'un ver de terre par exemple, ou via une galerie de campagnol.

35 - Le charbon prélevé à la base $(1,65 \mathrm{~m})$ des colluvions agricoles, sur le versant sud du Bickenberg est pour sa part daté de $1250 \pm 50 \mathrm{BP}$, soit un âge calibré compris entre 1261-1168, 1160-1136 ou 1107-1092 BP. En simplifiant, il date d'entre 690 et 860 de notre ère. Le début de la mise en culture du Bickenberg est à rapporter à la fin des temps mérovingiens, ou aux époques carolingiennes. Cet âge est cohérent avec deux datations obtenues sur des charbons prélevés sous un pierrier du Strangenberg : $1000 \pm 110$ (900 à 1180 de notre ère en âge calibré) et $1130 \pm 50$ BP (780 à 980 de notre ère) (LitaudonJouve, à paraître).

36 Comme nous l'avons expliqué dans les méthodes, l'âge d'enfouissement théorique des paléosols est calculé en soustrayant le TMR des matières organiques du sol témoin au TMR des matières organiques des paléosols conservés sous les pierriers. Les calculs donnent ainsi un âge théorique d'enfouissement de $596 \pm 42,408 \pm 27$ (Pr 1) et $628 \pm 42$ 
ans (Pr 2). La première et la troisième de ces valeurs ne sont pas statistiquement différentes, contrairement à la deuxième. Mais le deuxième prélèvement a été effectué à proximité de la bordure du pierrier, et les matières organiques ont été rajeunies par l'activité biologique du sol. Cette activité est d'ailleurs importante, comme on peut s'en rendre compte à la lecture des âges des matières organiques interstitielles, qui ont un TMR très faible, même dans les niveaux profonds du pierrier Pr 1 . Il est très vraisemblable que même dans les palésols conservés sous les pierriers la matière organique a été rajeunie par l'incorporation d'une fraction de carbone moderne. C'est la raison pour laquelle il vaut mieux considérer le résultat des datations comme un âge minimum. On peut ainsi affirmer que les pierriers ont au moins de 550 à 670 ans d'âge, c'est-à-dire qu'ils datent au plus tard des années 1330/1450 AD. Pour sa part, la mise en évidence d'une forte érosion agricole circa 690-860 AD marque le début des labours sur le Bickenberg. On peut ainsi admettre que les pierriers ont été érigés à une date encore imprécise, mais nécessairement comprise entre la fin du VII et le milieu du $\mathrm{XV}^{\mathrm{e}}$ siècle de notre ère.

\section{Discussion autour d'un scenario de reconstitution paysagère}

La datation par les charbons de bois de la base des colluvions agricoles de la rue SaintMarc, qui reposent en discordance directement au contact d'une formation périglaciaire, donne le début de la mise en culture du Bickenberg. Cette date est comprise entre 690 et 860 de notre ère. On notera la convergence entre cette date et celles de la création des grandes abbayes voisines, comme Murbach (728), Munster (vers 660) et le couvent Saint-Marc, situé à $4 \mathrm{~km}$ au NNE d'Osenbach, qui aurait été fondé en 676 par Dagobert II. Cette convergence ne nous parait pas anodine, et il nous semble vraisemblable que la mise en culture du Bickenberg soit une conséquence directe de la création de ce couvent, qui possédait de nombreux biens dans le secteur et qui a fortement contribué comme les autres institutions du même type, au développement des vallées vosgiennes. Au vu de l'abondance des charbons de bois, tant dans ces colluvions que dans les sols conservés sous les pierriers de l'ensemble des collines sèches, il semble que cette mise en culture se soit produite après le défrichement de zones largement couvertes de forêts de chênes (Litaudon-Jouve, en cours).

Il n'est pas possible de relier directement l'épierrement qui a abouti à la formation des murgers à cette première phase agricole, les datations étant trop imprécises. Nous pouvons en revanche cerner la période dans laquelle s'inscrit obligatoirement l'érection des Steinrudeln: elle est comprise entre cette date haute de 680-860 AD et le terminus ante quem de 1330/1450. A priori, deux époques sont à privilégier dans cette tranche d'âge : le VIII siècle, époque de la création et du développement des grandes abbayes, et la période qui s'étend grosso modo de 1050 à 1150, et qui a correspondu à un fort essor démographique dont témoignent les très nombreuses églises romanes qui s'érigent ou s'agrandissent dans un rayon de 10-15 km autour d'Osenbach. Les églises d'Osenbach, Wintzfelden, Rouffach, Soultzmatt, Pfaffenheim, Gueberschwihr, Guebwiller, Murbach, Merxheim, Gundolsheim, Reguisheim, Meyenheim, Lautenbach datent quasiment toutes de cette époque, densité étonnante au regard même de la dimension modeste de certaines de ces localités. Cette deuxième période semble même 
plus probable que la première. En effet, les sols des collines sèches sont très ingrats à travailler : peu profonds voire squelettiques, très caillouteux, à réserves hydriques très limitées tant pour des raisons climatiques qu'édaphiques, il fallait une motivation importante pour justifier un travail de l'ampleur de l'érection des pierriers alors que de bons sols agricoles ne manquaient pas. Dans cette optique, les densités de population du VIII ${ }^{e}$ siècle sont faibles, les historiens s'accordant pour considérer que les mouvements monastiques de cette époque s'apparentent à une véritable colonisation des collines sous-vosgiennes et vallées (Juillard, 1953a; Nonn, 1983), où les traces d'habitats historiques sont rares avant cette époque. En revanche, la densité des églises romanes et villages témoigne d'une forte densité de population aux XI et XII ${ }^{e}$ siècles, dont une conséquence a pu être la nécessité d'intensifier la production agricole, même sur des sols pauvres. Un autre argument en ce sens est la datation des charbons prélevés sous les pierriers du Strangenberg. Si on accepte l'hypothèse que leur érection est contemporaine de celle des pierriers du Bickenberg, l'âge donné par le plus jeune de ces charbons plaide pour les $\mathrm{XI}^{e}$ ou XII ${ }^{e}$ siècles. On a toutefois vu, sur le Bickenberg, que des charbons pouvaient s'introduire par voie biologique assez tardivement sous les pierriers. Aussi convient-il de rester prudent par rapport à cet argument. Ce n'est qu'avec des datations supplémentaires et une étude plus approfondie des colluvions de la rue Saint-Marc que ces hypothèses pourront être affinées.

Par ailleurs, il semble que l'érection des murgers a été réalisée d'une traite, en un laps de temps relativement bref. Trois arguments plaident en ce sens. Le premier est la cohérence de la structure d'ensemble des murgers du Bickenberg, qui apparaît peu compatible avec des épierrements individuels, s'échelonnant sur une longue période. En particulier, la continuité du grand murger qui ceinture tout le Bickenberg offre l'image d'un plan d'aménagement global du lieu. Cette impression est largement confortée par la récurrence de certaines distances, dont notamment, au pied du versant Est, la répétition 9 fois de suite d'une distance de $54 \mathrm{~m}$ encore observable dans le parcellaire actuel. Ceci suggère un lotissement par l'autorité possédant les biens (le couvent Saint-Marc ?). Enfin, l'absence totale de discontinuités stratigraphiques et de paléosols au sein des pierriers est peu compatible avec des épierrements très échelonnés dans le temps. L'ensemble de ces éléments nous font émettre l'hypothèse que les pierriers résultent d'un travail collectif, sous l'égide d'un "maître d'oeuvre" décidé à mettre en valeur ce secteur en un laps de temps très bref. Cette hypothèse peut étonner au vu des grandes dimensions de ces pierriers, et dans un contexte culturel qui tend souvent à privilégier la continuité séculaire du labeur agricole dans le façonnement des paysages. Elle n'a toutefois rien d'impossible, comme le montre la simulation qui suit.

Le volume actuel des Steinrudeln du Bickenberg représente environ $25000 \mathrm{~m}^{3}$ (Bernardy, 1998). En partant de l'hypothèse que par le jeu des destructions ultérieures (utilisation pour le drainage des murets de vigne, comme on peut l'observer à différents endroits; pour l'empierrement de chemins ou de routes; pour le drainage de bâtiments), le volume initial était du double, ce qui est sans doute surestimé, on arrive à un volume total de l'ordre de $50000 \mathrm{~m}^{3}$. Si on y ajoute les conditions suivantes: utilisation de hottes d'une contenance de 501 (soit environ $65 \mathrm{~kg}$ de pierres, en tenant compte du volume de vide), 5 heures de travail par jour (le reste de la journée étant occupé par des tâches incontournables comme la traite), et un nombre de 3 allers et retours par heure en moyenne entre le plateau à épierrer et les murgers, on arrive à un ordre de grandeur de 67000 journées de travail nécessaires pour édifier les murgers. 
Pour une communauté d'une centaine de travailleurs, cela représente donc 670 journées de travail, soit 2,5 années complètes en tenant compte des jours fériés, ou bien 10 années de travail si celui-ci est simplement réparti sur les mois d'hiver. Ce chiffre d'une centaine de travailleurs n'a rien d'invraisemblable dans un village doté très tôt d'une église en pierre, et cela d'autant plus que, comme le signale olivier de Serres (1600), ce type de travail n'était pas réservé aux hommes. Femmes, enfants, vieillards pouvaient y contribuer, avec des charges certes moins lourdes, mais diminuant d'autant le temps de travail total. Ce calcul ne donne certes que des ordres de grandeurs: nous ignorons la taille effective de la communauté qui a participé à ce travail collectif. Nous ignorons également tout du temps de travail journalier, du volume exact des hottes utilisées. Les représentations de hottes médiévales en osier, comme celle figurant sur l'enluminure symbolisant le mois de février dans le Rustican de Pierre de Crescens (XVe siècle : représentée in Braudel, 1986) montrent toutefois que le volume était proche de $501 . .$. et qu'on les portait bien pleines! On ne peut de plus exclure l'utilisation d'animaux de bât, comme des ânes. Cependant, même si nous ne pouvons donner que des ordres de grandeur, on se rend bien compte que ce type de travail était tout à fait réalisable en un laps de temps relativement court, compris entre 1 et 20 ans pour prendre des bornes larges.

41 Par ailleurs, il n'est pas certain que le Bickenberg ait été labouré pendant une longue durée. Le caractère ingrat des sols a sans doute limité dans le temps cette utilisation, dont on ne retrouve d'ailleurs aucune trace écrite dans les quelques archives historiques et cartographiques qui nous sont parvenues. Si nos hypothèses concernant l'âge des pierriers ou l'aspect collectif et rapide du travail dont ils sont les témoins sont vraisemblables, les tenants et aboutissants mêmes du défrichement du Bickenberg, de sa mise en culture, de l'érection des murgers nous échappent encore.

\section{Conclusion}

Pour ponctuel et partiel qu'il soit, ce travail apporte bien des précisions quant à la dynamique de paysages agraires encore peu étudiés de l'Alsace, ceux des collines sousvosgiennes. Il montre en particulier que si dans cette région l'openfield domine, d'autres types d'occupation des sols ont également eu cours aux époques médiévales. L'Alsace n'a pas échappé aux paysages d'enclos, parfois qualifiés de «bocages lithiques " bien connus dans de nombreuses régions françaises. On ne peut citer ici de façon exhaustive tous les travaux qui leur ont été consacrés. On consultera, par exemple, Zadora-Rio (1991) ou Lewuillon (1991) pour des synthèses, ou encore un site internet (www.pierreseche.com) consacré à ces structures. En Alsace, celles-ci ne sont pas cantonnées aux seules collines sèches, où elles sont assez bien préservées, sans doute grâce à la principale utilisation ultérieure des collines, le pâturage extensif, qui n'a pas nécessité de réaménagement important. A Osenbach même, il en existe quelques lambeaux à l'Ouest du village, dans le secteur actuellement viticole du village, au lieu-dit appelé Steinfeld (champs de pierres). Deux pierriers linéaires existent aussi sur la colline gréseuse située juste à l'est du Bickenberg. Et très récemment, Denni (2002) en a décrit dans le cadre de l'étude paysagère du Wurmberg, une colline calcaire située à proximité d'Obernai. Dans ce secteur, ils prennent le nom de Steinrotteln, dans lequel on reconnaîtra sans peine l'équivalent bas-rhinois de Steinrudeln. Les habitants du secteur différencient selon Denni (2002) les Langrotteln des Rundrotteln, c'est à dire 
les pierriers linéaires des pierriers circulaires. On notera la ressemblance entre ces termes et celui de rottern, défricher en vieil allemand, à l'origine de nombreux toponymes (Rotthüttel, Rothenbrand, Hohrod, Roderen, Rottbach, Rodern,...). Cette ressemblance n'est peut-être qu'une coïncidence phonétique, mais une étude linguistique plus approfondie pourrait être intéressante. Ce qui est certain, c'est que ces Steinrudeln qui sortent un peu de l'oubli grâce à cette étude, et qui nous plongent au cœur même de l'origine des villages des collines sous-vosgiennes, mériteraient une plus grande attention, en particulier une approche archéologique directe que nous n'avons pas encore pu développer.

\section{BIBLIOGRAPHIE}

L'étude de Bernardy (1998), a été financée par la municipalité d'Osenbach et les établissements CEREST. Nous en remercions chaleureusement Mr. L. Burklen, Maire d'Osenbach, F. Brender, adjoint chargé de l'environnement, toute la municipalité d'Osenbach, et Mr. R. Hurth, PDG des établissements CEREST. Les datations ultérieures ont été financées par le programme PNSE (Programme National sur les Sols et l'Erosion) du CNRS/INSU. Nos remerciements vont également à Mr. J. Ehrhart, Président de la Société d'Histoire de Rouffach, à L. Olive, responsable du cadastre de Guebwiller, à Y. Michelin, historien et géographe des paysages (ENITAH Clermont-Ferrand) et G. Bischoff, professeur d'Histoire médiévale à l'Université Marc Bloch de Strasbourg pour leurs conseils, à W. Fleck du bureau géologique régional de Fribourg qui a traduit le résumé en allemand, à E. Hiniger pour les documents qu'ils nous a prêtés, à C. Kaiser, gérante du camping et J. Neunlist, secrétaire générale de mairie, pour l'accueil qu'elles ont réservé à L. Bernardy. Nous remercions enfin tous les habitants d'Osenbach qui ont apporté leur concours amusé et intéressé à cette étude, dont les premiers résultats leur ont été livrés "en primeur" en 1998 sous forme de conférence publique.

BALESDENT J. et Guillet B. (1982). - Les datations par le ${ }^{14} \mathrm{C}$ des matières organiques des sols. Contribution à l'étude de l'humification et du renouvellement des substances humiques. Science du Sol, 20, 2, 93-112.

BERNARDY L. (1998). - Etude du parcellaire du Bickenberg (Commune d'Osenbach, Haut-Rhin). Mémoire de maîtrise de Géographie, CEREG/Université L. Pasteur, Strasbourg, 66 p.

BIEHLER E. (1965). - Les voies romaines de la région de Colmar. Mémoire DES Histoire, Univ. Strasbourg, $66 \mathrm{p}$.

BOEHLER J.M., (1967). - De la reconstruction à la révolution agricole. L'évolution de la vie rurale dans quelques villages du Kochersberg, 1660-1836. Mémoire DES Histoire, Univ. Strasbourg, 400 p.

BoYÉ P. (1903). - Les Hautes-Chaumes des Vosges. Berger-Levrault, Nancy, 432 p.

BRAUDEl F. (1986). - L'identité de la France. T. 1 : espace et histoire, 1re partie. Arthaud-Flammarion, $368 \mathrm{p}$.

Dion R. (1959). - Histoire de la vigne et du vin en France des origines au XIXe siècle. Paris, 768 p. 
CALlot H.J. (1980). - La plaine d'Alsace. Modelé agraire et parcellaire. Publ. Univ. Nancy II, 338 p.

D6 C. (2002). - Etude des aménagements ruraux du Wurmberg. Aspects historiques, physiques et paysagers. ULP Strasbourg, Fac. géographie, Mém. Géographie physique, 87 p.

DufouRnET J. (1995). - Les Très Riches Heures du Duc de Berry. Bibliothèque de l'Image, 80 p.

GARNIER E. (1993). - L'homme et son milieu. Le massif du Grand Ventron, à travers les âges. Univ. Fr. Comté, Fac. Lettres de Besançon, PNRBV, Décembre 1993, 64 p.

GARNIER E. (1998). - Jalons pour une histoire de l'environnement : la réserve naturelle du FrankenthalMissheimle. Labo Sciences Historiques, Univ. Besançon/PNRBV, Munster, 59 p.

GARNIER E. (2000). - Les forêts des Vosges méridionales à l'époque moderne: des espaces forestiers éclatés et convoités (T2). Thèse Doct. d'Hist. (nouveau régime), Univ. Fr.-Comté, Fac. Lettres et Sc. Hum., $775 \mathrm{p}$.

GARNIER E. (2002). - Plans anciens et reconstitution paysagère. Le système montagnard vosgien (XVI ${ }^{\mathrm{e}}-\mathrm{XVIII}{ }^{\mathrm{e}}$ siècle). Histoire et Sociétés rurales, 17, 123-152.

GUILLET B. (1979). - Etude du renouvellement des matières organiques des sols par les radioisotopes (14C). In : M. Bonneau et B. Souchier (éds.), Pédologie, t.2, Constituants et propriétés des sols. Masson, Paris, p. 210-226.

HATTJ.J. (1959). - Traces de centuriations en Alsace. Rev. Archéol. Est, 10, 302-308.

HERTZog Dr. A. (1900). - Die Entwicklung und Organisation des Elsäsischer Weinbaues von der frühesten Zeit bis zu heutigen Tagen. In : Festschrift zum 19. Deutschen Weinbau-Congress in Colmar, 22-26 Sept. 1900, in-16, p. 49-108.

Hoff M. (1978). - Les collines calcaires sous-vosgiennes. Bull.Soc. Ind. Mulhouse, 77, 2, 17-33.

KOERNER W., BENOIT M., DAMBRINE E. et DUPOUEY J.L. (1999). - Influence des anciennes pratiques agricoles sur la végétation et les sols des forêts reboisées dans le massif vosgien. Rev. For. Fr., 51, 2, 231-238.

KoERner W., DAmbrine E., Dupouey J.L. et Benoit M. (1999). $\mathrm{d}^{15} \mathrm{~N}$ of forest soil and understorey vegetation reflect the former agriculture land use. Oecologia, 121, 421-425.

JACOB J.C. (1984). - Landes de Westhalten. Bull. Soc. Indust. Mulhouse, 795, 4, 3.

JuILLARD E. (1953a). - La vie rurale dans la plaine de Basse-Alsace. Publ. Inst. Hautes Etudes Alsaciennes, IX, Strasbourg, $582 \mathrm{p}$.

JUILlARD E. (1953b). - Formes de structure parcellaire dans la plaine d'Alsace. Bull. Ass. Géogr. français, 72-77.

JuillaRd E. (1959). - Traces de centuriation en Alsace. Rev. Archéol. Est, 10, 298-301.

LEWUiLlon S. (1991). - Les murs de pierre sèche en milieu rural. In : J. Guilaine (éd.), Pour une archéologie agraire. Armand Colin, Paris, p. 193-221.

LitAUdDON-JouvE C., en cours. Origine et dynamique des pelouses sèches du secteur de Rouffach. Applications à leur gestion conservatoire. Thèse doct. Univ. Louis Pasteur, Strasbourg (soutenance prévue fin 2004).

NonN H. (1983). - Déboisements. In : Encyclopédie de l'Alsace, t. 4, Publitotal, Strasbourg, p. 2284-2287.

PAULP. (1983). - Climat. In : Encyclopédie de l'Alsace, vol. 3. Publitotal, Strasbourg, p. 1769-1777. 
Sell Y., Berchtold J.P., CAllot H., Hoff M., Gall J.C. et Walter J.M. (1998). - L'Alsace et les Vosges. Géologie, milieux naturels, flore et faune. Delachaux et Niestlé, Lausanne, 352 p.

SERRES O. de (1600, ré-édition 1996). - Le théâtre d'agriculture et mesnage des champs. Réédition Thesaurus, Actes Sud, Arles, 1463 p.

SitTig C. (1934). - La vie d'une commune alsacienne, Vendenheim, depuis le début du XIX ${ }^{\mathrm{e}}$ siècle. Rev. d'Alsace, p. 359-382.

SitTLER C. et MAROCKE R. (1981). - Terroirs et vins d'Alsace. Sci. Géol. Bull., 34, 3, 147-182 + annexes, $3^{\circ}$ édition.

THÉOBALD N. (1977). - Carte géologique de la France à 1/50000. Feuille Neuf-Brisach/Obersaasheim. BRGM, Orléans, Notice, 43 p. +1 carte h.t.

TRICART J. et Usselmann P. (1967). - Feuille géomorphologique Neuf-Brisach 1/2. Notice CNRS RCP n 77. Rev. Géomorph. dyn., 17, 1, 10-20.

TURLOT J.P. (1978). - Les orchidées des collines sous-vosgiennes. Bull. Soc. Indust. Mulhouse, 770 (1978-2), 35-40.

VonNA A. (2003). - La cartographie des pierriers comme méthode d'approche d'un territoire. Collines du Zinnkoepflé, du Strangenberg et du Bollenberg. Mémoire de maîtrise de géographie physique, ULP, Strasbourg, $128 \mathrm{p}$.

WURTZ M. (1976). - Centuriations romaines en Moyenne-Alsace. Rev. Archéol. Est et du Centre-Est, $27,1-2,149-170$.

ZADORA-RIO E. (1991). - Les terroirs médiévaux dans le Nord et le Nord-Ouest de l'Europe. In : J. Guilaine (éd.), Pour une archéologie agraire. Armand Colin, Paris, p. 165-191.

\section{RÉSUMÉS}

De nombreux murgers résultant de l'épierrement de sols calcaires s'étendent sur les collines sèches des environs de Rouffach (Haut-Rhin). Ces murgers, appelés localement Steinrudeln forment des éléments complexes plus ou moins réticulés, de type "paysage d'enclos", structurant un ancien parcellaire. L'étude détaillée de ces structures sur le Bickenberg à Osenbach a permis de reconnaître le caractère médiéval de ce parcellaire, dont l'origine est comprise entre les années 690/860 et 1330/1450. En fait, il est vraisemblable qu'il date des $\mathrm{XI}^{\mathrm{e}}$ ou XII ${ }^{\mathrm{e}}$ siècles. Il n'a pas été possible d'en préciser davantage l'âge, en raison de l'absence totale d'archives historiques liées à ces structures, ce qui nous a obligé à utiliser des approches paléoenvironnementales. Bien qu'imprécise, cette attribution chronologique n'en constitue pas moins un progrès par rapport aux connaissances antérieures, puisque des défrichements étaient évoqués depuis le Néolithique, tandis que la tradition locale voyait dans les murgers le résultat des activités agricoles du XIX ${ }^{\mathrm{e}}$ siècle. Ces Steinrudeln, édifiés sur des terres ingrates peu propices à une utilisation agricole intensive sont sans doute liés à la pression démographique importante des $\mathrm{XI}^{\mathrm{e}}$ et $\mathrm{XII}^{\mathrm{e}}$ siècle, dont les nombreuses églises romanes du secteur proche constituent un témoignage indirect. Ils forment un type de paysage agricole peu décrit et original en Alsace, région associée à l'openfield.

Numerous stone accumulations resulting from field clearing of stones can be observed on some calcareous hills covered by a xeric grass vegetation near Rouffach (Haut-Rhin, France). These stone acuumulations are locally called Steinrudeln. They form a complex net structure corresponding to an ancient field pattern. The study of these structures on the Bickenberg, an 
hill located in Osenbach, allowed to date this field pattern from the medieval times. The stone cuumulations were built between 690/860 and 1330/1450 AD, without more chronological precisions. However, they probably date from the $\mathrm{XI}^{\mathrm{e}}$ or $\mathrm{XII}^{\mathrm{e}}$ centuries, a period corresponding to a high demographic level. They form an original kind of landscape in Alsace, which is generally considered as an openfield area.

Bei Rouffach (Haut-Rhin, Frankreich) sind auf Kalkhügeln mit xerophiler Grasvegetation Anhäufungen von Lesesteinen weit verbreitet. Diese Steinanreicherungen werden mit dem Lokalbegriff "Steinrudeln» bezeichnet. Sie bilden eine komplexe Netzstruktur, welche die frühere Feldeinteilung widerspiegelt. Die Untersuchungen auf dem Bickenberg bei Osenbach ergaben eine mittelalterliche Entstehung dieser Parzellenstruktur. Die Steinanhäufungen wurden zwischen 690/860 und 1330/1450 n. Chr. angelegt, wobei sich die Datierung nicht weiter präzisieren lässt. Aufgrund der hohen Bevölkerungsdichte im 11. und 12. Jahrhundert sind sie wahrscheinlich in diesem Zeitraum entstanden. Sie zeugen von einer ursprünglich offenen Landschaftsform (openfield) im Elsass.

\section{INDEX}

Mots-clés : Alsace, Bickenberg, colline sèche calcaire, épierrement, murger, parcellaire médiéval

Schlüsselwörter : Bickenberg, Elsaß, mittelalterliche Feldeinteilung, Steinanreicherung, xerophile Kalkhügel

Keywords : Alsace, Bickenberg, clearing of field of stones, medieval field pattern, stone accumulation, xeric calcareous hill

\section{AUTEURS}

\section{DOMINIQUE SCHWARTZ}

Université L. Pasteur, Faculté de Géographie, 3, rue de l'Argonne, 67083 Strasbourg

\section{LAURENCE BERNARDY}

Université L. Pasteur, Faculté de Géographie, 3, rue de l'Argonne, 67083 Strasbourg

\section{CLAIRE JOUVE-LITAUDON}

Université L. Pasteur, Faculté de Géographie, 3, rue de l'Argonne, 67083 Strasbourg / ENGEES, 1 quai Koch, 67082 Strasbourg ; act. DDAF18, Centre administratif Condé, 18013, Bourges

\section{ANNE VONNA}

Université L. Pasteur, Faculté de Géographie, 3, rue de l’Argonne, 67083 Strasbourg 